\title{
Abordagem neurocognitiva de processos atencionais envolvidos na aprendizagem mediada por mapas conceituais
}

Kleyfton Soares da Silva kley.soares@hotmail.com 0000-0002-8526-961X Instituto Federal Goiano, Campos Belos, Goiás, Brasil.

Laerte Silva da Fonseca laerte.fonseca@uol.com.br 0000-0002-0215-0606

Instituto Federal de Sergipe, Aracaju, Sergipe, Brasil.

Paulo Rogerio Miranda Correia prmc@usp.br

0000-0003-2419-7103

Universidade de São Paulo, São Paulo, São Paulo, Brasil.

\begin{abstract}
RESUMO
O engajamento atencional do aluno durante a aprendizagem parece ser o principal objetivo a ser alcançado quando se está elaborando um material instrucional. O mapeamento conceitual tem sido difundido com uma técnica promissora para a aquisição e representação de ideias, revelando-se como uma alternativa pedagógica útil no cotidiano escolar. O objetivo deste trabalho é apresentar subsídios teóricos nos campos da neurociência cognitiva, psicologia cognitiva e educação para justificar, a partir do funcionamento cerebral da atenção, estratégias pedagógicas que exploram o uso de mapas conceituais. Um quadro de referência foi produzido com indicações de mapas conceituais que levam em consideração o fator "atenção" no processo de aprendizagem, como o mapa com erros, com lacunas, com figuras e com cores/contrastes. Baseada nas noções de mecanismos atencionais e processamento da informação da Teoria da Carga Cognitiva, uma proposta de construção de mapas conceituais foi apresentada para exemplificar a aplicação da literatura discutida.
\end{abstract}

PALAVRAS-CHAVE: Atenção. Mapas conceituais. Neurociência cognitiva e educação. 


\section{INTRODUÇÃO}

Estratégias de ensino têm ganhado cada vez mais possibilidades de intervenção em sala de aula para alcançar objetivos de aprendizagem específicos (HATTIE; DONOGHUE, 2016). Como resultado, uma variedade de métodos surge a todo instante na tentativa de minimizar dificuldades de aprendizagem associadas, principalmente, à natureza do conteúdo. No Brasil, muito se discute sobre a necessidade de práticas pedagógicas visando à aprendizagem significativa, tendo em vista a possibilidade de tomar os conhecimentos prévios dos alunos como ponto de partida para a construção de novos conhecimentos.

A ideia da aprendizagem significativa repousa na possibilidade da integração de conhecimentos novos a uma estrutura cognitiva do sujeito já consolidada ou em consolidação, de onde surgem novas compreensões e, consequentemente, aprimoramento de concepções (AUSUBEL, 2000). Não é uma aprendizagem permanente no sentido literal, mas plástica, contextual, podendo ser modificada ao longo do tempo ou esquecida.

Embora a questão do conhecimento prévio venha sendo tratada como o fator principal a se considerar durante a elaboração de estratégias pedagógicas, o entendimento do funcionamento cerebral de funções cognitivas como a "atenção" pode abrir portas para a elaboração de estratégias mais eficazes, uma vez que o potencial de produção de aprendizagem do aluno depende da sua atenção e do seu engajamento cognitivo.

Enquanto ferramenta pedagógica, o mapa conceitual (MC) tem sido importante tanto para fins de pesquisa, quanto para a aprendizagem rotineira em sala de aula (ZARPELON; RESENDE; PINHEIRO, 2015). Se construído pelo próprio aluno, o MC tende a revelar a organização hierárquica dos conceitos aprendidos e suas relações. Esse é o tipo de MC mais incentivado na escola, mas não tem sido o mais satisfatório para alguns contextos devido ao tempo investido na sua produção, avaliação e devolutiva (CORREIA; AGUIAR, 2016). Além disso, a falta de treinamento dos estudantes pode levar à construção de MCs inapropriados.

Por outro lado, se o MC é concebido pelo professor, espera-se que o aprendiz reflita sobre as suas ideias segundo uma estrutura espacial e hierárquica prédefinida. Esse tipo de $M C$ traz vantagens quanto à necessidade de treinamento somente pelo professor; à maior possibilidade de clareza e precisão hierárquica dos conceitos; à familiarização pelos aprendizes de um MC adequado; à diversidade de avaliações de desempenho que podem ser empregadas; ao tempo ganho para investimento em outras tarefas (CORREIA; CABRAL; AGUIAR, 2016).

Quais seriam, portanto, as possibilidades de representação visuoespacial de um determinado conteúdo por meio de um MC manipulado pelo professor? Para responder a essa pergunta, recorreu-se às noções de mapas conceituais apresentadas por Novak (2010) e a princípios oriundos da psicologia cognitiva e neurociência cognitiva que colocam a "atenção" como principal função cognitiva associada à aprendizagem.

Inicialmente, apresentamos a noção de mapeamento conceitual e suas aplicações, destacando elementos importantes que abrem espaço para a discussão sobre processos atencionais. Após a abordagem de conceitos subjacentes a tais processos, discutimos o papel da atenção na aprendizagem por meio de MCs, levando em consideração que os benefícios dessa ferramenta dependem do foco 
dispensado pelo estudante nos processos cognitivos desencadeados a partir dos estímulos visuais presentes nos MCs. Por fim, introduzimos conceitos da Teoria da Carga Cognitiva (SWELLER, 2011) para informar alguns procedimentos instrucionais que o professor deve considerar ao trabalhar com MCs.

\section{NOÇÕES E APLICAÇÕES DO MAPA CONCEITUAL}

A técnica de mapeamento conceitual concebida na década de 1970 por Novak e colaboradores serviu, a priori, como um instrumento de pesquisa para compreender as concepções de crianças sobre determinados tópicos de ciências (NOVAK, 2010). A Figura 1 apresenta um MC, que é um organizador gráfico que representa as relações entre os conceitos envolvidos numa estrutura de conhecimento, geralmente evocada a partir de uma pergunta focal. É importante destacar que todo conhecimento é construído a partir de conceitos e proposições, pois não se pode validar ou invalidar fatos isolados sem uma estrutura linguística convencionada. Nesse sentido, as proposições formam unidades de significados e são combinações de dois ou mais conceitos que afirmam sobre o tema representado (NOVAK, 2010).

Figura 1 - Mapa conceitual que responde à pergunta focal “Quais são os principais elementos de um MC e suas relações?

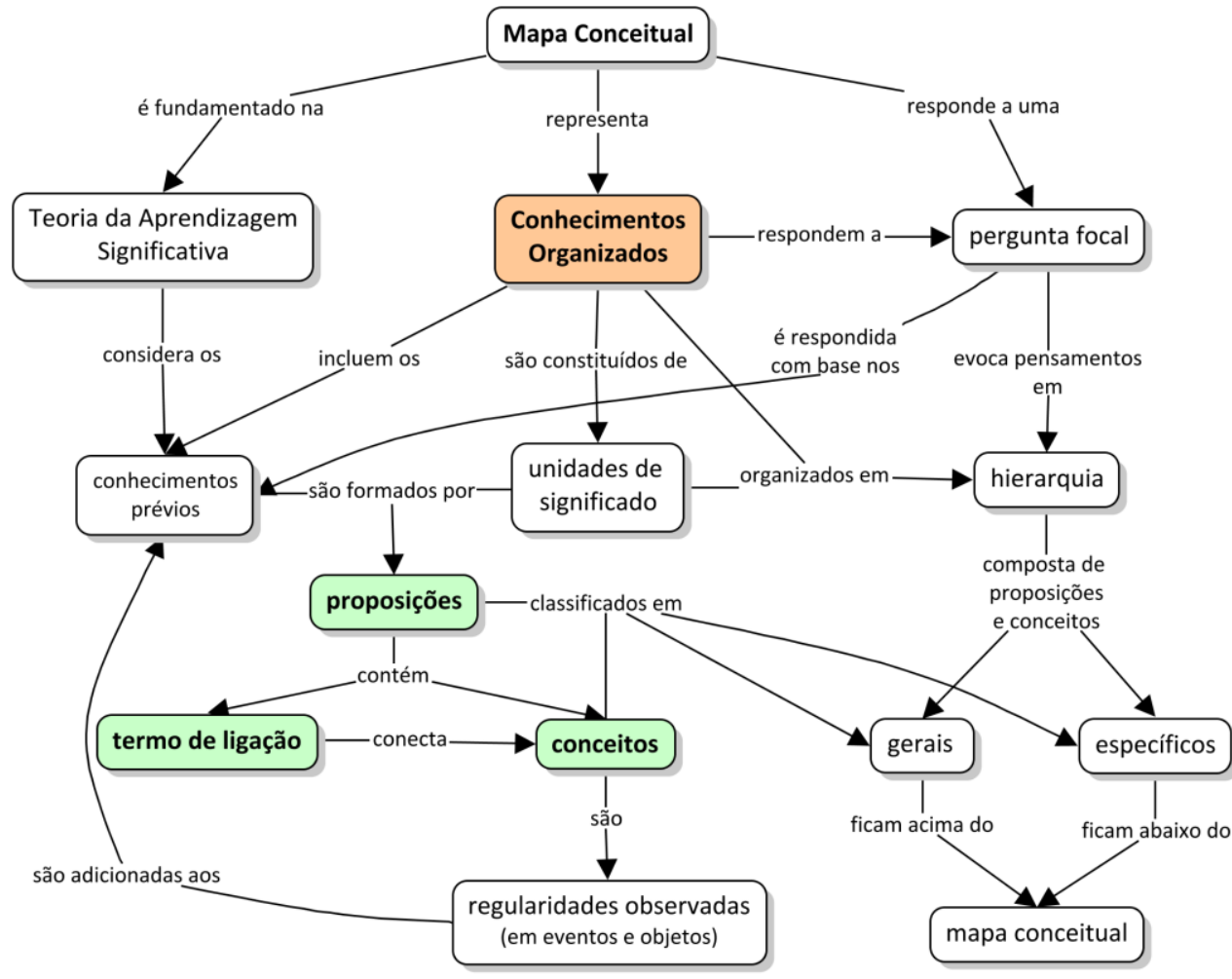

Fonte: Autores (2020).

Atualmente, os MCs têm auxiliado estudantes e educadores a buscarem a aprendizagem significativa. Com efeito, a base teórica que subsidia os estudos de Novak é a Teoria da Aprendizagem Significativa (AUSUBEL, 2000), ao considerar 
1. A aprendizagem significativa - em contraste à aprendizagem mecânica - é necessária para a conceitualização;

2. A nova aprendizagem deve se ancorar em conceitos e proposições já existentes na estrutura cognitiva do aprendiz;

3. O aluno deve ser incentivado a optar pela aprendizagem significativa;

4. Materiais concretos adequados são necessários para aprender conceitos abstratos, juntamente, com instruções didáticas apropriadas;

5. A aprendizagem é altamente idiossincrática e progride com o tempo;

6. A aprendizagem significativa de alta qualidade leva à construção de estruturas conceituais e proposicionais bem integradas que facilitam a nova aprendizagem e a resolução criativa de problemas (CAÑAS; NOVAK, 2006, p. 2).

Dada a característica criativa da produção de mapas conceituais (MCs), não há um modelo correto ou perfeito, na verdade, existem alguns elementos essenciais para a exposição de ideias segundo um raciocínio coerente. Novak (2010) explica que o mapeamento conceitual tende a se desenrolar hierarquicamente, com os conceitos mais gerais e inclusivos no topo do mapa e os mais específicos e menos inclusivos para baixo. Os novos conceitos a serem incorporados em uma estrutura cognitiva são retidos com mais robustez quando o sujeito já tem conhecimentos prévios, sendo mais fácil partir do geral e ir para os seus detalhes (diferenciação progressiva), do que partir de vários detalhes para determinar o conceito geral (reconciliação integrativa) (AUSUBEL, 2000).

Os MCs têm sido utilizado, principalmente, para melhorar a aprendizagem do aluno que o produz em sala de aula (ZARPELON; RESENDE; PINHEIRO, 2015); como instrumento de avaliação formativa ou de pesquisa científica (DANTAS; SILVA; BORGES, 2018). Frequentemente, alguns materiais instrucionais trazem uma síntese em forma de $M C$ do que foi ou será abordado no material. Porém, o que se tem visto é uma disseminação da ideia de aprender ou sintetizar conteúdos através de mapas conceituais sem considerar fundamentos oriundos de pesquisas da área (CAÑAS; NOVAK, 2006).

A falta de treinamento na técnica de mapeamento conceitual se apresenta como um problema. Novak, Cañas e Reiska (2015) argumentam que a clareza de um MC depende do seu conteúdo e da sua estrutura gráfica. Para tentar minimizar o efeito desconcertante do uso de mapeamento conceitual sem o devido cuidado, Aguiar e Correia (2013) discutiram alguns parâmetros de referência para a construção adequada de MCs com base na necessidade de estabelecimento de clareza semântica das proposições, pergunta focal, organização hierárquica dos conceitos e revisões contínuas. Em outro momento, Aguiar e Correia (2017) propuseram uma sequência de treinamentos que vai da representação do conhecimento (escolha dos conceitos e sua organização hierárquica) a sua modelagem (estabelecimento de proposições válidas e relações entre os conceitos).

E quanto à avaliação dos MCs? Notadamente, a diversidade de representação dos organizadores gráficos chama a atenção dos educadores que precisam encontrar estratégias de avaliação. Dessa forma, a estrutura (organização gráfica) 
e conteúdo (conjunto de proposições) são os principais indicadores de qualidade de um MC. Ao sugerirem critérios para a avaliação qualitativa de MCs, Novak, Cañas e Reiska (2015) enfatizam que um MC hierarquicamente estruturado pressupõe uma estrutura cognitiva organizada (AUSUBEL, 2000). Relativamente ao conteúdo, os autores reforçam a importância da relevância dos conceitos, da qualidade dos termos de ligação e da validade das proposições.

Embora uma acentuada preocupação se volte à qualidade estrutural e conceitual do MC, poucas pesquisas (BALLEY, 2005; SANTOS et al., 2016) têm direcionado o foco para questões mais específicas que levem em consideração o funcionamento cerebral da aprendizagem e sua relação com as estratégias pedagógicas que sugerem o uso de MCs. Os pressupostos teóricos da Teoria da Aprendizagem Significativa embasam o funcionamento da aprendizagem em seus desdobramentos cognitivos e apontam as relações da aquisição e retenção de novos conhecimentos com os materiais instrucionais, mas não esclarecem - do ponto de vista neurocognitivo - o funcionamento da memória e as variáveis pelas quais as informações externas são interpretadas pelo sujeito, como cor, forma, via de entrada (auditiva, olfativa, gustativa, visual, tátil).

A especificação das relações entre os materiais instrucionais e o funcionamento cognitivo tem sido cada vez mais necessária, uma vez que pesquisas educacionais vêm revelando as variações no desempenho da aprendizagem quando se opta por uma ou outra abordagem metodológica e, também, por uma ou outra ferramenta pedagógica.

\section{PROCESSOS ATENCIONAIS}

A atenção é um dos pilares da aprendizagem, pois sem ela a facilitação da passagem das informações ao longo das sinapses com a finalidade de formar memórias duradouras não seria possível (COSENZA; GUERRA, 2011). Segundo Gazzaniga e colaboradores (2006, p. 265), "a atenção é um mecanismo cerebral cognitivo que possibilita alguém processar informações, pensamentos ou ações relevantes, enquanto ignora outros irrelevantes ou dispersivos".

Resultados de pesquisas em psicologia sobre a atenção levaram cientistas a classificá-la basicamente em atenção voluntária e automática (LENT, 2010). A utilização de métodos de eletroencefalografia e neuroimagiologia tem reforçado o envolvimento de estruturas cerebrais específicas para a atenção voluntária focalização de estímulos de forma consciente - e para a atenção reflexa, quando a orientação é reflexa para novos estímulos no ambiente, como som, luz, movimento, contraste.

Fiori (2008) ressalta que, em 1994, os psicólogos Posner e Raichle identificaram uma série de operações mentais relacionadas ao ato de prestar atenção a eventos visuoespaciais: a captação da atenção por um estímulo (processo automático) eleva o estado de alerta do sujeito; há um processo de localização espacial e focalização do alvo; há um desengajamento atencional da região espacial como consequência da criação de expectativa de um novo alvo e uma nova localização; há uma busca pela nova localização e focalização do novo alvo (processo controlado).

A especialização de sistemas atencionais para processos automáticos e controlados sugere que algumas áreas do cérebro são responsáveis pela 
seletividade e manutenção (controle) da atenção. Esse tipo de esclarecimento é importante porque o processamento da atenção decorre dos estímulos recebidos pelos órgãos sensoriais e seu controle é dependente do que já existe na memória do indivíduo (GAZZANIGA et al., 2006).

São discutidos dois tipos de atenção. A atenção seletiva se refere aos mecanismos cerebrais incumbidos de selecionar as informações mais importantes e ignorar os estímulos distraidores (GAZZANIGA et al., 2006). Esse fenômeno é facilmente percebido quando alguém que está predeterminado (ação voluntária) a localizar um determinado alvo sensibiliza a sua atenção para as características do objeto e o encontra rapidamente mesmo em meio ao "caos".

A atenção concentrada é a que parece mais preocupar os educadores, pois é demandada, sobretudo, durante a aprendizagem. Nesse contexto, há uma intensificação das atividades neuronais responsáveis pela percepção e compreensão dos sistemas de linguagem, uma vez que há uma maior sensibilização da rede anterior da atenção para atividades de compreensão de palavras (MATLIN, 2004). Dessa forma, a atenção concentrada está em ação quando há a focalização do alvo e a tentativa de decodificação dos seus significados.

A Figura 2 mostra as duas principais áreas cerebrais - rede posterior e anterior - que regulam os mecanismos atencionais segundo o modelo de Posner e Raichle. A rede posterior da atenção compreende o córtex parietal e está envolvido em atividades de orientação espacial. É a área cortical mais ativada quando se está em busca de um alvo visual. Já a rede anterior da atenção que envolve parte do lobo frontal responde à focalização da atenção em símbolos linguísticos, como as palavras. É nessa mesma área que os neurônios especializados em inibir respostas automáticas a estímulos são sensibilizados (POSNER; PETERSEN, 1990).

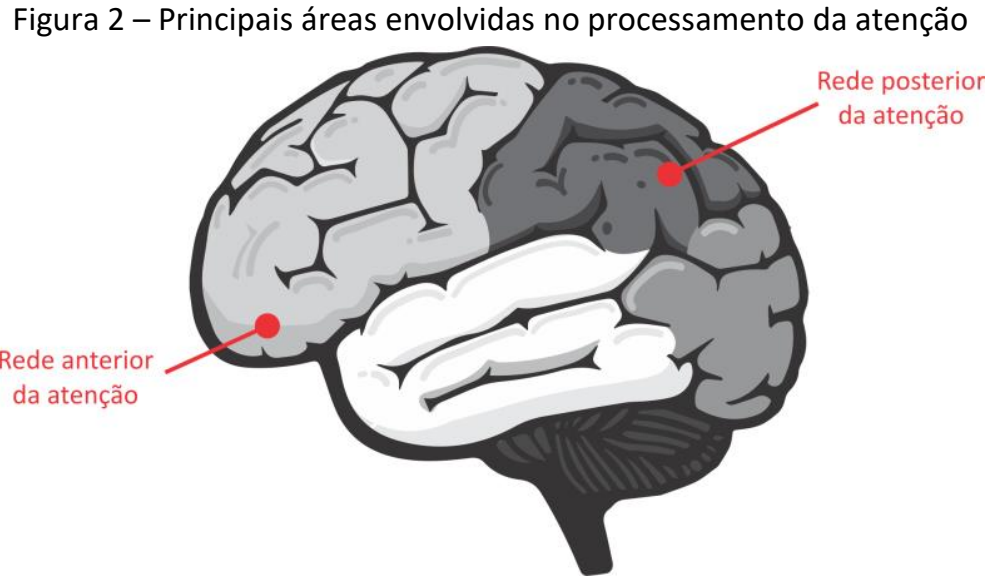

Fonte: Autores (2020), adaptado de iconicbestiary/Freepik.

Genericamente, pode-se dizer que a operacionalização da atenção se dá segundo dois processos: bottom-up, que decorre da percepção de estímulos sensoriais que acabam por guiar a focalização do alvo, e top-down, cujo processamento da informação não depende exclusivamente da percepção dos estímulos sensoriais, mas sim da expectativa criada previamente e responsável por dirigir o foco da atenção (STEVENS; BAVELIER, 2011). O processsamento bottomup é guiado pelo estímulo. Ou seja, o estímulo influencia a nossa percepção, direcionando a nossa consciência cognitiva para o objeto. Em contraste, o 
processamento top-down usa os conhecimentos prévios e as expectativas para influenciar a nossa percepção. Nesse caso, as expectativas baseadas no que o sujeito já conhece moldam a percepção daquilo que é alvo da atenção.

De forma simplificada, um elemento de contraste presente em um MC pode direcionar o foco da atenção do leitor de forma automática, partindo da percepção do estímulo visual e alcançando processos cognitivos associados ao conteúdo observado (processamento bottom-up). Por outro lado, se considerarmos que um aluno observa um MC a partir da sua pergunta focal, processos cognitivos relativos aos conhecimentos prévios do aluno podem entrar em ação antes mesmo de o foco ser dirigido para o MC. Cria-se, nesse caso, uma expectativa e o foco é direcionado em função do objetivo do leitor (processamento top-down), que seria o de encontrar estímulos visuais que levem a uma resposta. Portanto, cabe o questionamento sobre como os princípios do funcionamento da atenção podem contribuir para o delineamento de estratégias pedagógicas envolvendo o MC.

\section{RELAÇÃO ENTRE A APRENDIZAGEM POR MAPAS CONCEITUAIS E OS PROCESSOS ATENCIONAIS}

Os MCs enquanto organizadores gráficos requisitam um alto grau de alerta atencional para que a mensagem seja processada no cérebro do leitor. Em linhas gerais, a ideia de funcionamento do $M C$ é compatível com os modelos de processamento das informações aludidos pelas pesquisas em neurociência cognitiva e psicologia cognitiva (SANTOS et al., 2016).

Os efeitos da produção de MC pelo aluno podem ser positivos, mas a construção de bons mapas por iniciantes é uma tarefa difícil e geralmente causa sobrecarga cognitiva (ACUNA; AYMES; LOPEZ MEDRANO, 2014). Dessa forma, o modelo de $M C$ tratado neste tópico é aquele criado pelo educador e disponibilizado ao aluno para mobilizar a aprendizagem inicial de um conteúdo, além de servir para organizar e avaliar o conhecimento previamente estudado. Segundo Correia, Cabral e Aguiar (2016), o uso do MC com essa finalidade traz vantagens em relação ao criado pelo próprio aluno porque enquanto o professor se preocupa em aprender a técnica de mapeamento, os alunos precisam somente ler o mapa e dedicar seus recursos cognitivos ao tema estudado.

\section{Mapa com erros}

O MC com erros (Figura 3) é uma importante ferramenta para analisar a capacidade dos estudantes em localizar relações conceituais inapropriadas (CORREIA; CABRAL; AGUIAR, 2016). Trata-se de um mapa criado pelo professor e estruturado visando à validação de proposições dentro de uma rede integrada de conceitos. Considerando as limitações da atenção, sobre esse tipo de mapa é relevante considerar: a qualidade e relevância dos erros conceituais; a quantidade de erros conceituais a serem incorporados; suas posições espaciais. 
Figura 3 - Exemplo da aplicação de erros em MC sobre propriedades da matéria. As marcações em vermelho mostram os erros que deverão ser encontrados e corrigidos pelos alunos, que recebem mapas em preto e branco

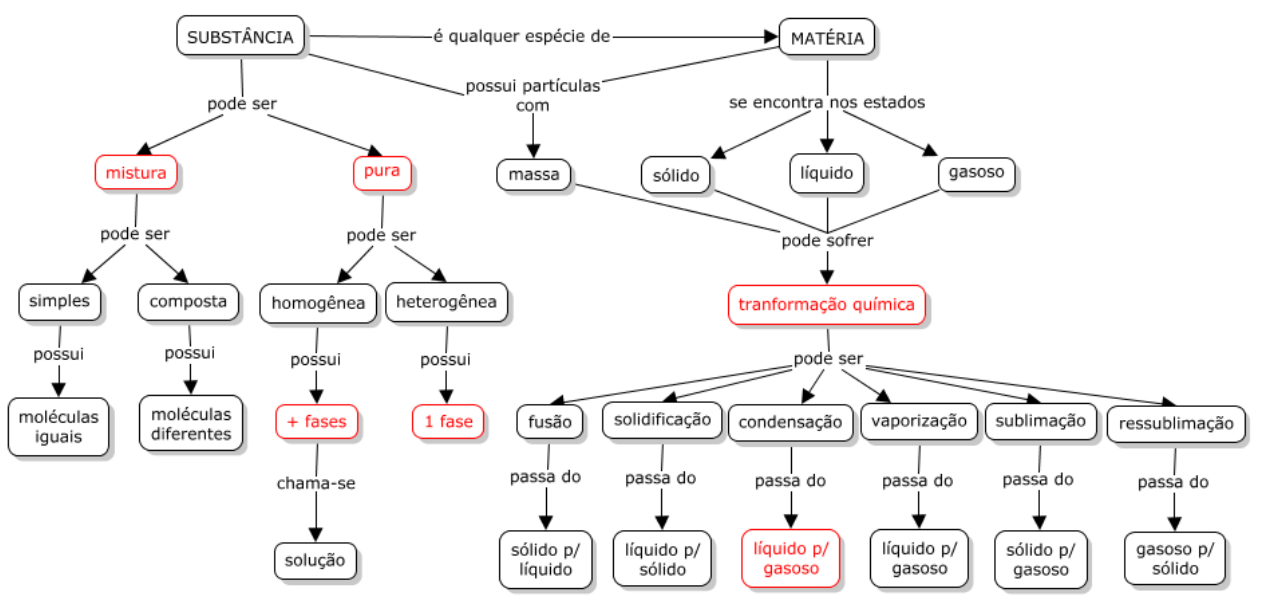

Fonte: Autores (2020).

Depois de concebido um mapa satisfatório em conteúdo e estrutura, sugerese pensar estrategicamente na incorporação dos erros conceituais de modo a incentivar a leitura integral do conteúdo. Vale salientar o cuidado que deve ser empregado na escolha e quantidade das proposições a serem evocadas, pois o emprego da atenção espacial associada à busca visual ao longo da estrutura do mapa, a atenção para os significados da linguagem verbal e a manutenção das informações pertinentes na memória de trabalho são atividades que requerem muito esforço das funções executivas, podendo levar a uma sobrecarga cognitiva (SWELLER, 2011).

O MC com erro parece funcionar como um detector de incoerências nos padrões de conhecimentos previamente estabelecidos em experiências cotidianas e escolares. Em tese publicada em 2011, Brockington (2011) mostrou que as respostas emocionais diante de interpretações de conceitos científicos dependem do nível de conhecimento do sujeito. Professores e alunos, por exemplo, reagem diferentemente durante a interpretação de conceitos científicos e alternativos. Ademais, o fator emocional é preponderantemente necessário para regular o nível de motivação e, consequentemente, de atenção durante a resolução de problemas.

Em mapeamento de regiões cerebrais ativadas durante a escuta de frases semanticamente e sintaticamente incongruentes, Friederici e colegas (2003) identificaram respostas cerebrais mais ativas nos lobos frontal e temporal. Uma série de experimentos permitiu sugerir que existem processos automáticos de compreensão da linguagem, de modo que quando o sujeito é surpreendido com frases semanticamente incorretas, como "o gato come cenoura", ou sintaticamente incorretas, como "o gato leite toma", ondas do potencial evocado são instantaneamente geradas, indicando que diferentes partes do cérebro se interconectam formando uma espécie de léxico mental (FIORI, 2008).

De modo análogo, esses fatos sugerem que diante de proposições incorretas, o aluno reage emocionalmente em função dos seus conhecimentos prévios, que, 
por seu turno, influenciam diretamente o engajamento atencional. O princípio \#8 - ou PEN \#8, de Psychology, Education, Neuroscience - organizado pelo Centro de Pesquisa em Ciências da Aprendizagem da Austrália ${ }^{1}$ traz evidências científicas de que o erro pode ser considerado uma falha produtiva (KAPUR, 2016).

Um aluno de alto desempenho ativa circuitos neurais ligados à atenção e memória, sugerindo que esse tipo de aluno se engaja em processos produtivos na procura de erros e soluções, enquanto o aluno de baixo desempenho ativa primordialmente circuitos de recompensa, buscando feedback positivo (DOWNAR; BHATT; MONTAGUE, 2011).

\section{Mapa com lacunas}

Uma estratégia voltada à captação da atenção para aspectos específicos do conteúdo pode ser a inclusão de lacunas para ser preenchidas, tanto no lugar de termos de ligação, quanto de conceitos (Figura 4). Ao ler um MC comum, o aluno pode prestar atenção nos conceitos e rapidamente supor uma ligação entre eles, como uma concepção do senso comum, sem necessariamente focar nos termos de ligação.

Figura 4 - Exemplo da aplicação de lacunas em MC sobre propriedades da matéria. As marcações em vermelho mostram as lacunas que deverão ser preenchidas alunos

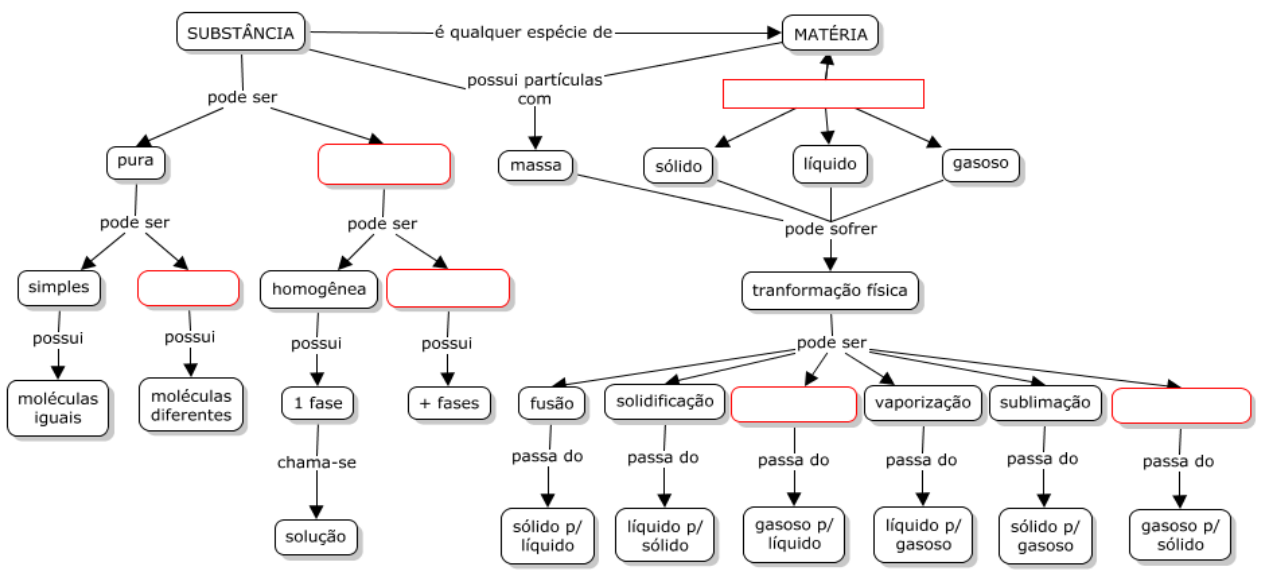

Fonte: Autores (2020).

A adição de lacunas nos termos de ligação, seguida de feedback, poderia minimizar esse efeito. Essa indicação condiz com os resultados que Bisra (2007) apontou quando verificou através da técnica de rastreamento ocular (eyetracking) que o leitor de MCs nem sempre fixa sua atenção nos termos de ligação, mas preponderantemente nos conceitos, revelando ser este um primeiro passo para conferir sentido aos MCs.

Outro fundamento para a indicação de MCs com lacunas tem relação com o princípio da pré-ativação de estratégias para guiar a aprendizagem (Princípio \#12, Centro de Pesquisa em Ciências da Aprendizagem da Austrália ${ }^{2}$ ), que consiste em estabelecer um quadro de conceitos para organizar o conteúdo aprendido ou a ser aprendido, de preferência, por meio da recordação ativa dos conhecimentos (VERDI et al, 1997). Nesse viés, na procura por respostas, o cérebro ativa previamente padrões de estímulo-resposta e processa as informações futuras com mais facilidade (SCHACTER; BUCKNER, 1998). 


\section{Mapa com figuras/ícones}

Estudos psicológicos e neurocientíficos (KANDEL et al., 2014) comprovaram a existência de mecanismos cerebrais específicos relativos à memória explícita - que é evocada conscientemente -, levando a identificação de dois subtipos dessa memória: a episódica e a semântica.

A memória episódica é basicamente a memória das experiências, eventos autobiográficos, série de fatos e cargas emocionais geradas nesses momentos. Já a memória semântica se refere a registros mais estruturados, de significado de símbolos verbais, de conceitos. Em geral, a memória semântica deriva da episódica, existe uma dialética entre elas, pois as experiências cotidianas vêm na maioria dos casos carregadas de significados (KANDEL et al., 2014).

A breve abordagem acerca da memória episódica e semântica abre espaço para a introdução de uma proposta que envolve o uso de figuras em MCs (Figura 5) com o objetivo de chamar a atenção do leitor para eventos específicos e potencialmente significativos. Nessa categoria de MCs, entra em jogo o papel do sistema de processamento da informação conhecido como duplo canal, que inclui os processamentos visual-pictórico e auditivo-verbal amplamente discutidos pela Teoria da Aprendizagem Multimídia (MAYER, 2014).

Figura 5 - Exemplo da aplicação de figuras/ícones em MC sobre propriedades da matéria. Os ícones mostram as possíveis disposições espaciais que deverão ser identificadas e associadas ao conteúdo

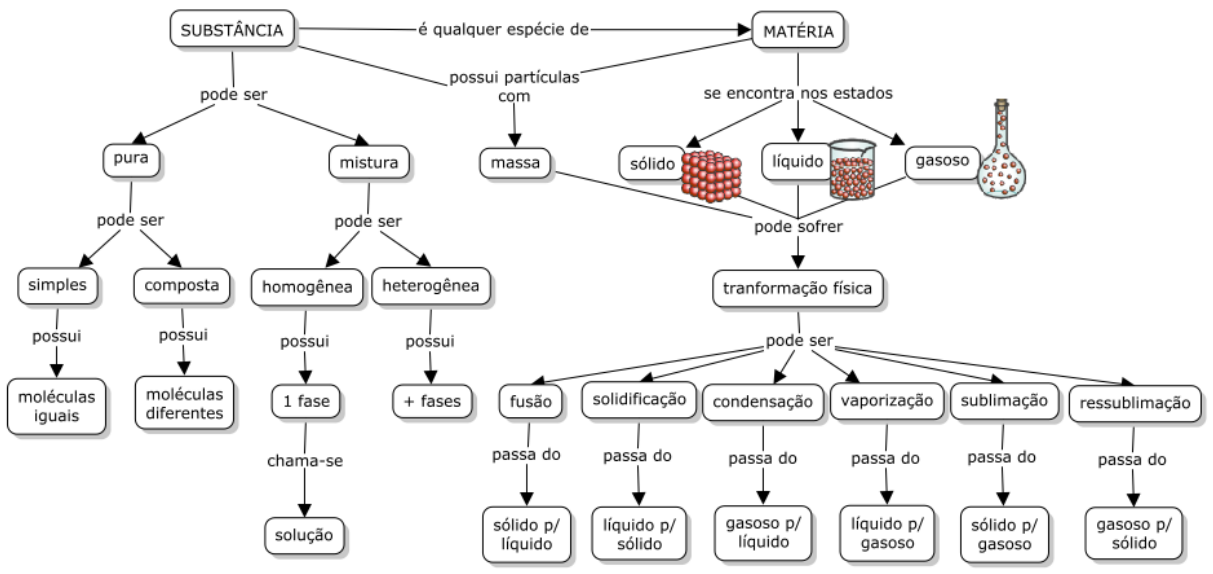

Fonte: Autores (2020).

Nessa perspectiva, materiais instrucionais que privilegiam imagens e textos/áudio são mais facilmente compreendidos e produzem memórias mais robustas (JOHNSON; ZATORRE, 2006). O fato de o cérebro interpretar os dois tipos de informação simultaneamente consiste na distinção de regiões cerebrais responsáveis pelo processamento de imagens e compreensão da linguagem escrita e falada (Princípio \#2, Centro de Pesquisa em Ciências da Aprendizagem da Austrália ${ }^{3}$.

Quanto ao MC, a memória episódica faria a composição ponto a ponto do momento da aprendizagem, como o registro das imagens ou ícones presentes e suas posições espaciais (direita, esquerda, acima, abaixo) associadas a grupos de conceitos. 


\section{Mapa com cores/contrastes}

Essa proposta surge com elementos indispensáveis para o direcionamento automático da atenção do estudante: o contraste e a novidade. Um dos princípios básicos da atenção é que não se consegue manter o foco em mais de uma coisa por vez (LENT, 2010). "É natural intuir que essa ação focalizadora só se torna possível porque conseguimos sensibilizar seletivamente um conjunto de neurônios de certas regiões cerebrais que executam a tarefa principal, inibindo as demais" (LENT, 2010, p. 631).

Nesse sentido, é recomendado guiar a atenção do leitor ao longo do MC por meio da marcação de informações que de alguma forma fazem parte de uma mesma categoria (Figura 6). Em se tratando de MC cíclico, uma pesquisa conduzida por Aguiar e Correia (2016) mostrou que a inserção de cores em grupos de conceitos mais próximos produziu um desempenho maior do que a utilização de MCs sem cores ou com indicadores numéricos para guiar a leitura.

Figura 6 - Exemplo da aplicação de cores/contrastes em MC sobre propriedades da matéria. As cores setorizam as categorias que complementam o conteúdo

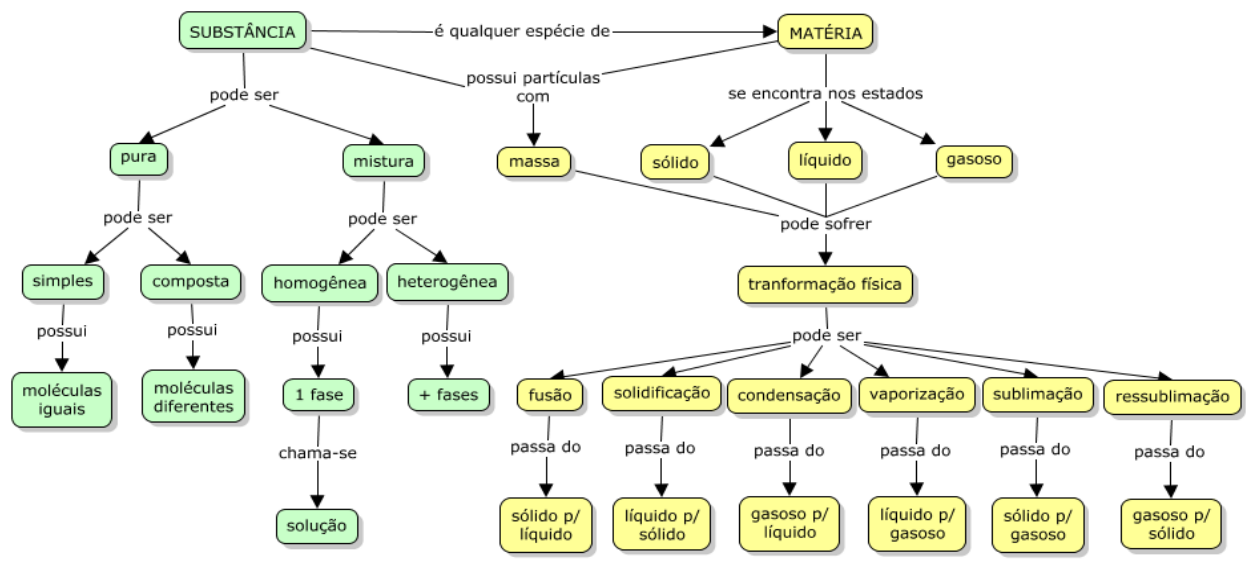

Fonte: Autores (2020).

A inserção de cores e de formas geométricas para diferenciar hierarquias ou grupos conceituais, bem como de tamanhos de fontes diferentes ou, ainda, aplicar negrito, pode gerar uma sensação de conforto durante a organização do conhecimento, considerando que a predição espacial dos alvos é um dos princípios de aprendizagem envolvidos na conceitualização (Princípio \#3, Centro de Pesquisa em Ciências da Aprendizagem da Austrália ${ }^{4}$ ).

A pesquisa de Summerfield e Egner (2009) sugere que o cérebro humano tende a organizar as informações em categorias, inclusive espaciais, para que a recordação seja facilitada, o que torna a dica visual um suporte para a localização de conceitos e proposições de maneira menos dispendiosa, já que expectativas relativas ao espaço são criadas e possivelmente fazem a leitura fluir.

\section{Quadro resumo dos tipos de mapas discutidos}

Como contribuição para o design instrucional visado pelo ensino, a abordagem teórica deste trabalho deu origem aos exemplos de estruturas de MCs mostrados no Quadro 1, trazendo indicações que comungam com as expectativas de aprendizagem do ponto de vista neurocognitivo, psicológico e educacional. 
Quadro 1 - Exemplos de mapas conceituais que consideram alguns princípios da atenção. Mostram apenas as estruturas que atendem às expectativas dos respectivos tipos de mapa. $\mathrm{TL}=$ termo de ligação

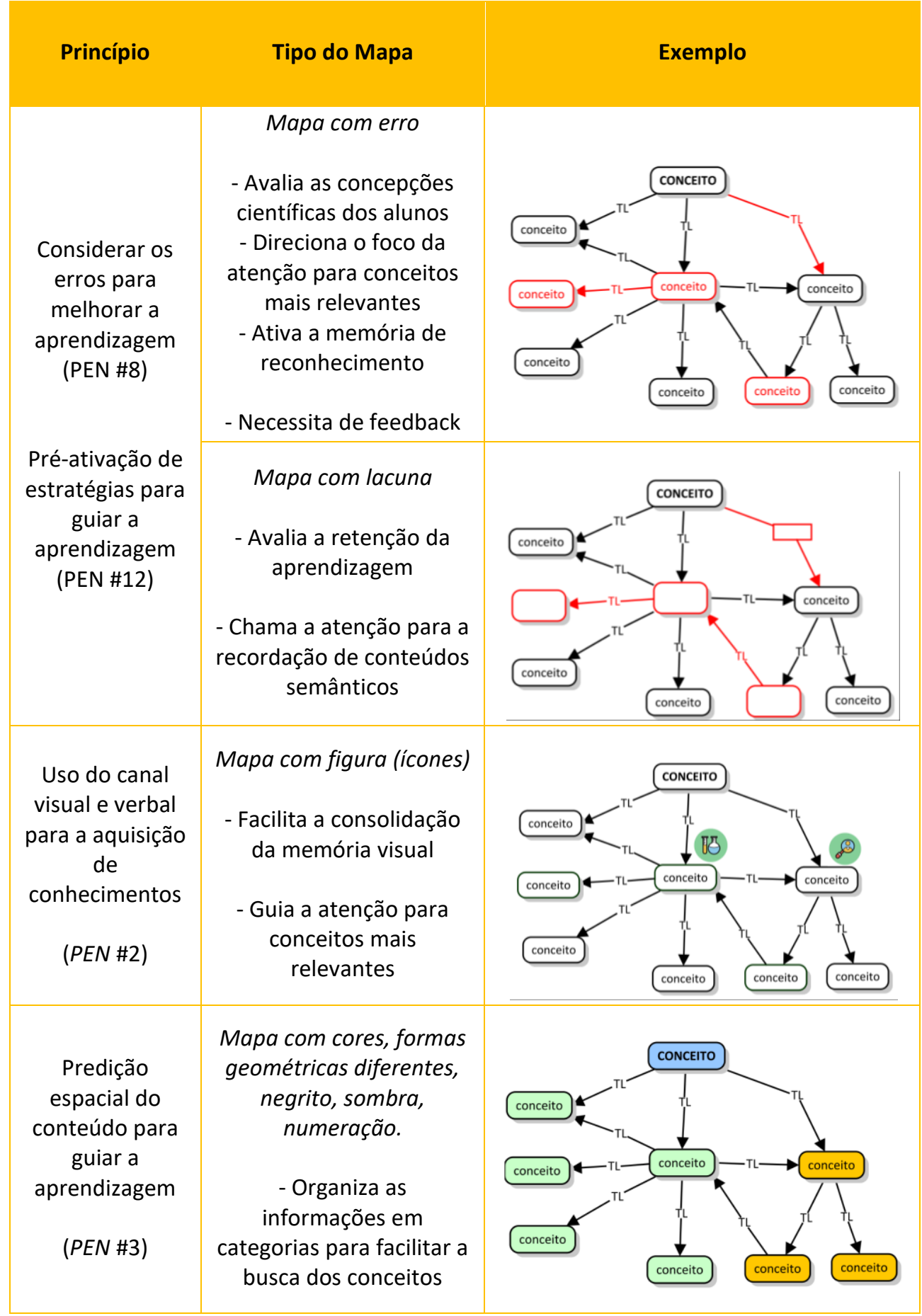

Fonte: Autores (2020).

\section{A TEORIA DA CARGA COGNITIVA E A ELABORAÇÃO DE MATERIAIS INSTRUCIONAIS}

A versatilidade de abordagens que têm o MC como ferramenta nos faz refletir sobre as implicações de estratégias textuais e gráficas usadas pelo mapeador. Além 
da preocupação com a organização proposicional hierárquica, a escolha dos conceitos e termos de ligação podem ser feitas com base nos conhecimentos prévios dos leitores, enquanto os elementos gráficos (cores, contrastes, figuras) podem ser incorporados ao mapa conceitual com o objetivo de direcionar o foco da atenção para áreas de interesse. Dessa forma, o conhecimento de mecanismos pelos quais a informação é processada no cérebro pode levar à elaboração de MCs mais efetivos.

Enquanto suporte para o design instrucional visado pelo ensino, a Teoria da Carga Cognitiva (TCC) leva em consideração a capacidade limitada de processamento da informação na memória de trabalho. Somente uma quantidade limitada de informação pode ser processada em qualquer momento, mas um número expressivo de informações organizadas pode ser processado e levar a resultados mais sofisticados (SWELLER, 2011).

Nessa perspectiva, a aprendizagem é resultado do processamento de novas informações na memória de trabalho (memória de curto prazo) que interagem com os esquemas já consolidados na memória de longo prazo, transformando-os em novos esquemas (SWELLER, 2011). A TCC considera que as novas informações são processadas na memória de trabalho (que é limitada) e articuladas com os esquemas da memória de longo prazo, em que o resultado deve ser o armazenamento de novos esquemas.

De acordo com a TCC, o gerenciamento de informações na memória de trabalho demanda cargas cognitivas de naturezas distintas:

Carga cognitiva intrínseca - tem relação com a complexidade do conteúdo a ser aprendido; quanto mais informações associadas a um dado conceito forem requisitadas para a compreensão desse conceito, maior será o esforço cognitivo empreendido.

Carga cognitiva extrínseca - tem relação com o formato da tarefa que organiza o conteúdo a ser ensinado; quanto mais elementos distraidores fizerem parte do procedimento instrucional, maior será o esforço cognitivo empreendido.

A complexidade do conteúdo e o nível de compreensão do estudante são determinantes para a carga cognitiva intrínseca, mas ela não pode ser modificada pelo fato de se expressar devido a esses dois fenômenos que são inalteráveis, quando fixados o conteúdo e o nível do estudante. A construção de um MC sobre o átomo por um aluno e outro por um professor de química, por exemplo, pode demandar cargas cognitivas intrínsecas diferentes porque os níveis de compreensão dos sujeitos podem ser também diferentes. A outra maneira de causar mudanças na carga cognitiva intrínseca seria alterando o conteúdo, o que influi, portanto, na sua complexidade (SWELLER, 2011).

A forma como os procedimentos instrucionais são apresentados é a principal fonte de recursos cognitivos a serem processados na memória de trabalho, constituindo-se na carga cognitiva extrínseca. Tal carga pode e deve ser gerenciada visando à sua redução para propiciar melhores condições de aprendizagem.

É com base na ideia de gerenciamento dos materiais instrucionais apresentada na TCC que propomos a utilização de mapas conceituais elaborados pelo professor para: a) reduzir a carga cognitiva extrínseca imposta pelas atividades de sala de aula, evitando, assim, sobrecarga cognitiva; b) aumentar a 
capacidade de elaboração conceitual, ou atividade mental, para construir e automatizar esquemas cognitivos.

\section{AS NOÇõES DA ATENÇÃO E DA CARGA COGNITIVA NA PRÁTICA}

As noções sobre os mecanismos da atenção e sobre o processamento da informação discutido na TCC se apresentam como bases para o planejamento, elaboração, implantação e avaliação da aplicação de mapas conceituais em sala de aula. Para tal, sugere-se a observação de dois procedimentos:

Avaliação do contexto de aprendizagem: a natureza do conteúdo a ser mapeado, o nível de compreensão dos estudantes e a definição dos objetivos de aprendizagem devem orientar a escolha do tipo de mapa conceitual a ser elaborado.

Definição de elementos de contraste: identificação de conceitos ou grupos de conceitos que podem servir de gatilho para provocar processos atencionais.

O primeiro procedimento se refere aos cuidados necessários para a elaboração de um mapa conceitual adequado. Não há regras para o uso de certo tipo de mapa, pois o mapa com erro, com lacunas ou com figuras pode ser usado na aula introdutória, de revisão ou avaliação. $O$ seu uso vai depender dos objetivos de aprendizagem do professor e dos conhecimentos prévios dos alunos. 0 importante é levar em consideração as noções de carga cognitiva intrínseca e extrínseca para propiciar experiências de aprendizagem significativas. Para a produção do mapa conceitual, recomenda-se o aplicativo computacional CmapTools $^{5}$ e a leitura das recomendações sobre como construir um bom mapa conceitual (AGUIAR; CORREIA, 2013).

O procedimento posterior tem a ver com a escolha do suporte gráfico para direcionar o foco da atenção do leitor. As noções sobre o funcionamento da atenção são importantes porque podem conduzir a boas configurações de mapas conceituais de modo a propiciar o engajamento atencional do estudante.

Quando não se tem tempo para avaliar e gerar feedback para o aluno, o mapa produzido por ele pode não ter cumprido seu papel. A Figura 7 mostra um exemplo de mapa conceitual construído por um aluno inexperiente (em mapeamento conceitual) do 2 ㅇ ano do Ensino Médio. 
Figura 7 - Mapa conceitual síntese de um texto sobre o descarte de pilhas e baterias. 0 aluno foi instruído, em 5 minutos, sobre como construir um mapa conceitual (noções de hierarquia, conceitos e termos de ligação)

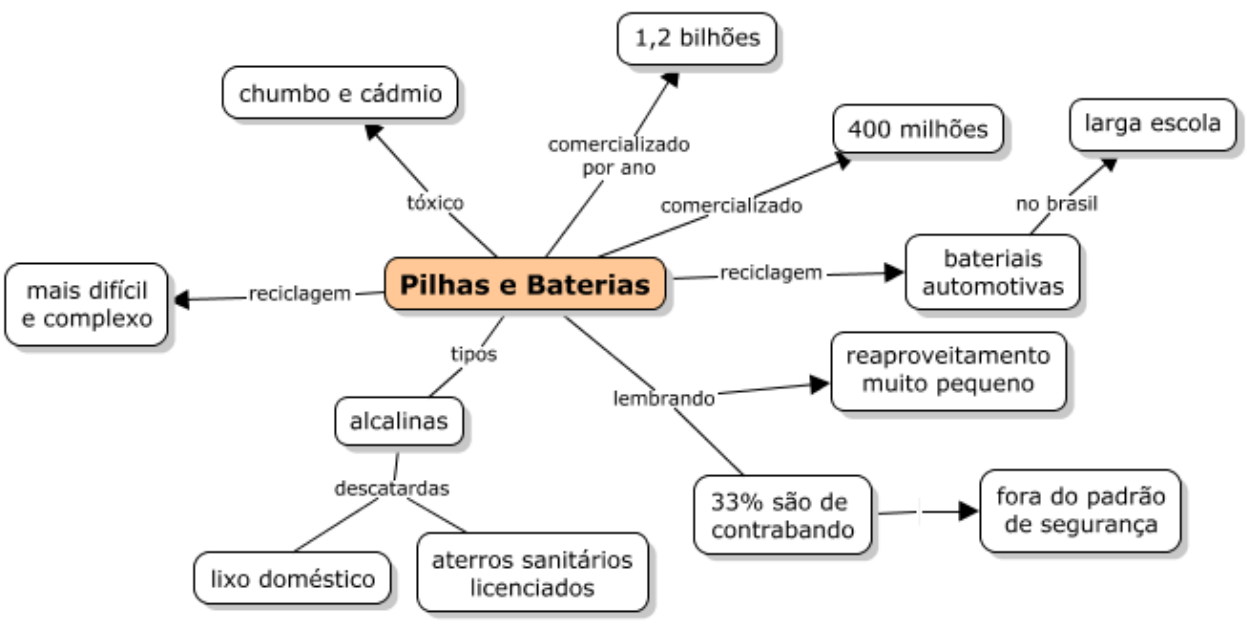

Fonte: A pesquisa (2020).

Nota-se que, devido à inexperiência do aluno, o mapa conceitual foi construído de maneira intuitiva, não atendendo à proposta de organização gráfica do conhecimento em níveis conceituais hierárquicos ou de inclusão de termos de ligação apropriados.

Enquanto sugestão do uso de mapa conceitual produzido pelo professor, analisemos a situação hipotética a seguir. Ao considerar a realização de uma atividade com mapa conceitual para trabalhar o conteúdo introdutório "propriedades da matéria", o professor avalia o contexto de aprendizagem e se certifica de que os alunos têm noção sobre partículas elementares e elemento químico. O conteúdo é pouco abstrato e se encontra num nível experimental facilmente observável, mas a preocupação se centra nos termos técnicos novos. $O$ objetivo é introduzir o tópico "transformações físicas da matéria", mas também revisar o tópico anterior "substância pura e mistura". Para isso, o professor prepara uma apresentação multimídia (projetor) com exemplos ilustrativos sobre transformações físicas no nível macroscópico. O tópico "substância" será revisado oralmente, apenas.

Nessas circunstâncias, uma sugestão (Figura 8) pode incluir estratégias do mapa com lacunas (para revisão de conteúdo) integrado ao mapa com figuras (para introdução de novos conceitos). 
Figura 8 - Mapa conceitual produzido para revisão e introdução de tópicos de química (substância e matéria, respectivamente)

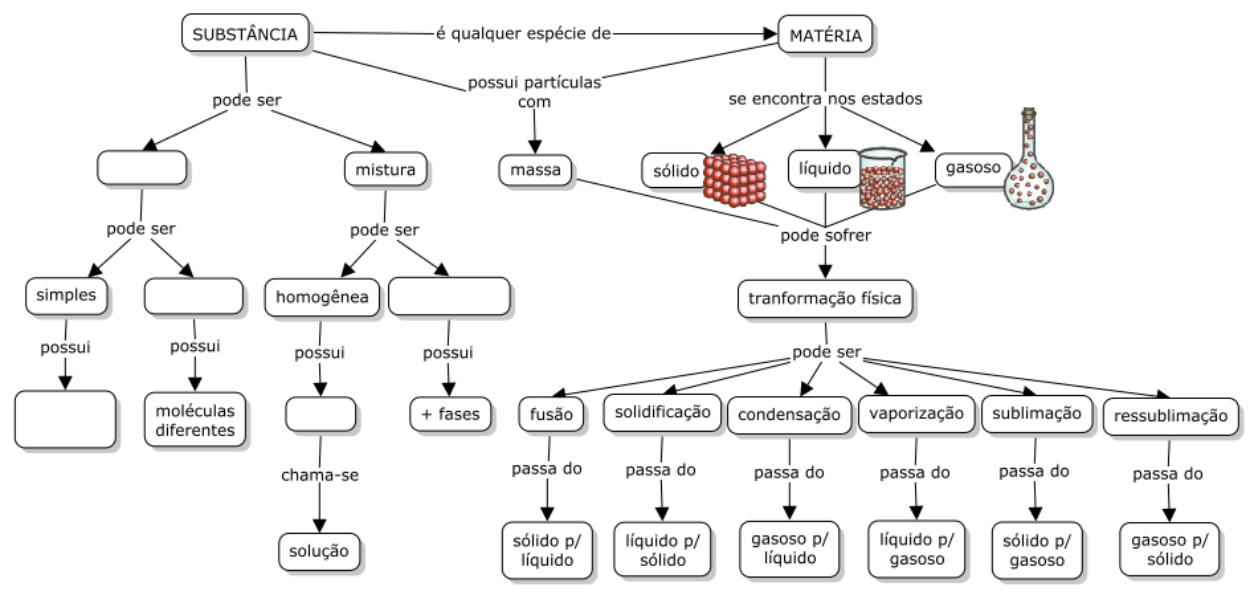

Fonte: Autores (2020).

Os elementos de contraste adicionados foram lacunas e figuras (ícones). As lacunas têm o papel de ativar memórias através da recordação ativa (VERDI et al, 1997) de conceitos mais próximos. Ao ler o conceito "mistura", por exemplo, há uma tendência em buscar informações nos arquivos de memória mais ou menos idênticas às codificadas no momento de aprendizagem (SCHACTER; BUCKNER, 1998). Dessa forma, se comparado com um mapa repleto de lacunas, essa estratégia minimiza a carga cognitiva extrínseca por dispor de elementos que ao mesmo tempo em que solicitam, também facilitam a recuperação de informações.

As lacunas são também destaques que possivelmente chamam a atenção do leitor por fugirem do padrão estrutural do mapa conceitual, mas sucedem a percepção das figuras, pois essas chamam mais atenção por possuírem informações salientes, levando a processos perceptivos imediatos. As figuras/ícones representam as informações verbais do mapa conceitual e pictóricas dos slides do professor de uma forma microscópica, trazendo novas informações importantes para serem decodificadas ao longo da aula, tais como a noção de calor e de agitação das partículas. Assim, a inclusão de conceitos e figuras espacialmente integrados pode contribuir para o aumento do desempenho do aluno (MAYER, 2014).

As figuras foram incluídas apenas para o conteúdo principal da aula, "transformações físicas", com o objetivo de: a) direcionar o foco da atenção de modo automático para permitir a seleção dos conceitos principais (processamento bottom-up); b) evocar memórias, de longo prazo, associadas à noção de movimento de partículas para a compreensão do conteúdo (processamento topdown) e sustentação da atenção na atividade; conduzir a focalização da atenção de forma voluntária para buscar outras respostas; c) deixar registros de memória visuoespacial para uma melhor transferência de conhecimentos (SUMMERFIELD; EGNER, 2009).

Nesse contexto hipotético, percebe-se que o mapa conceitual aparece para complementar os recursos de aprendizagem, não repetindo, necessariamente, as informações de outra forma. Os slides preparados pelo professor contêm exemplos ilustrativos, enquanto o mapa conceitual contém proposições que, em conjunto, narram o conteúdo, além de trazer, estrategicamente, figuras que 
definem outros conceitos não apresentados (calor, agitação das partículas). Para a revisão de "substância" o professor poderia sugerir uma discussão em dupla, seguido de feedback e explicações dos alunos sobre suas respostas.

Para a introdução de "transformações físicas" o professor poderia iniciar com uma contextualização; pedir para os alunos explicarem o mapa conceitual entre eles; levantar as dúvidas quanto aos conceitos novos; apresentar os slides com os exemplos; pedir para os alunos explicarem novamente suas compreensões.

Portanto, o exemplo dado serve como um convite à reflexão e implementação de estratégias pedagógicas baseadas nas noções de processamento da informação com ênfase nos mecanismos atencionais.

\section{CONSIDERAÇÕES FINAIS}

A abordagem teórica deste trabalho deu origem a exemplos de estruturas de mapas conceituais constituídos de elementos gráficos que tendem a regular os processos atencionais e propiciar melhores condições de aprendizagem. 0 objetivo foi apresentar teorias que justificam o planejamento, elaboração e aplicação de mapas conceituais com erros, lacunas, figuras/ícones e setorização de conteúdo com cores/contrastes. Hoje, algumas propostas de livros didáticos já trazem organizadores gráficos prontos no final dos capítulos, demonstrando que essa alternativa (mapa produzido pelo professor/especialista) tem sido aplicada e pode ser aprimorada.

Saber elaborar e gerenciar materiais instrucionais de forma que o aluno direcione a atenção para o alvo e permaneça focado na tarefa pode ser a chave para um ensino de qualidade. Cabe salientar que a leitura do mapa é um processo que requisita a atenção concentrada e precisa ser bem estruturado para ter o efeito esperado. Deve-se, ainda, evitar a sobrecarrega cognitiva devido à saturação dos esquemas gráficos com informações visuais desnecessárias e/ou confusas. 


\title{
NEUROCOGNITIVE APPROACH OF ATTENTIONAL PROCESSES INVOLVED IN LEARNING MEDIATED BY CONCEPTUAL MAPS
}

\begin{abstract}
The student's attentional engagement during learning seems to be the main goal to be achieved when developing instructional material. The concept mapping technique has been widely diffused for the acquisition and expression of ideas, proving to be a frequent pedagogical alternative in school routine. The aim of this research was to seek theoretical subsidies in the fields of cognitive neuroscience, cognitive psychology and education to justify, based on the cognitive functioning of attention, pedagogical strategies that make use of concept maps. A frame of reference was produced with indications of potential conceptual maps that take into account the factor "attention" in the learning process, such as map with error, with gap, with figure and with color. Based on the notions of attention mechanisms and information processing of the Cognitive Load Theory, a proposal for the construction of conceptual maps was presented to exemplify the application of the literature discussed.
\end{abstract}

KEYWORDS: Attention. Concept maps. Cognitive neuroscience and education. 


\section{AGRADECIMENTOS}

O presente trabalho foi realizado com apoio da Coordenação de Aperfeiçoamento de Pessoal de Nível Superior - Brasil (CAPES) - Código de Financiamento 001.

\section{NOTAS}

1 PEN Principle \#12 - Pre-activate strategies to guide learning. Disponível em: <https://www.slrc.org.au/pen-principal-12/>

2 PEN Principle \#2 - Visual images and spoken word mix well. Disponível em: <https://www.slrc.org.au/pen-2-visual-images-spoken-word-mix-well/>

3 PEN Principle \#3 - Spatial predictability guides attention. Disponível em: <https://www.slrc.org.au/pen-3-spatial-predictability-guides-attention/>

4 PEN Principle \#8 - Embrace error to improve learning. Disponível em: <https://www.slrc.org.au/pen-principle-8-embrace-error-improve-learning/>

50 download pode ser feito gratuitamente em: <https://cmap.ihmc.us/>.

\section{REFERÊNCIAS}

ACUNA, S. R.; AYMES, G. L.; LOPEZ MEDRANO, C. S. Fostering Multimedia Learning with Collaborative Concept Mapping: The Effect of Cognitive Aid on Performance and on collaboration. International Journal of Higher Education, v. 3, n. 2, p. 141-152, 2014.

AGUIAR, J. G.; CORREIA, P. R. M. Como fazer bons mapas conceituais? Estabelecendo parâmetros de referências e propondo atividades de treinamento. Revista Brasileira de Pesquisa em Educação em Ciências, v. 13, n. 2, p. 141-157, 2013.

AGUIAR, J. G.; CORREIA, P. R. M. From representing to modelling knowledge: Proposing a two-step training for excellence in concept mapping. Knowledge Management \& E-Learning, v. 9, n. 3, p. 366-379, 2017.

AGUIAR, J. G.; CORREIA, P. R. M. Using concept maps as instructional materials to foster the understanding of the atomic model and matter-energy interaction. Chemistry Education Research and Practice, n. 17, p. 756-765, 2016.

AUSUBEL, D.P. The acquisition and retention of knowledge: a cognitive view. Dordrecht, Kluwer Academic Pubishers, 2000. 
BALLEY, C. A. Concept mapping: A neuro-scientific approach. In: Midwest research-to-practice conference in adult, continuing, and community education. Anais... University of Wisconsin-Milwaukee, Milwaukee, WI, 2005.

BISRA, K. How learners visually navigate concept maps: an analysis of eye movements. 2010. Dissertação (Mestrado em Educação) - Simon Fraser University, Vancouver, Canadá, 2010.

BROCKINGTON, G. Neurociência e educação: investigando o papel da emoção na aquisição e uso do conhecimento científico. 2011. P. 202. Tese (Doutorado em Educação) - Universidade de São Paulo, São Paulo, 2011.

CAÑAS, A. J.; NOVAK, J. D. Re-Examining the foundations for effective use of concept maps. In: International Conference on Concept Mapping, 2, 2006. Anais... Costa Rica, 2006.

CAÑAS, A. J.; NOVAK, J. D.; REISKA, P. How good is my concept map? Am I a good Cmapper? Knowledge Management \& E-Learning, v. 7, n. 1, p. 6-19, 2015.

CORREIA, P. R. M.; AGUIAR, J. G. Um treinamento em duas etapas visando à certificação de mapeadores excelentes: da representação à modelagem de conhecimento. In: Innovating with Concept Mapping. 2016. Springer International Publishing.

CORREIA, P. R. M. ; CABRAL, G. C. P. ; AGUIAR, J. G. Cmaps with errors: Why not? comparing two cmap-based assessment tasks to evaluate conceptual understanding. In: International Conference on Concept Mapping, 7, 2016. Anais... New York: Springer, v. 1, p. 1-5, 2016.

COSENZA, R. M., GUERRA, L. B. Neurociência e educação: como o cérebro aprende. Porto Alegre: Artmed, 2011.

DANTAS, M. P.; SILVA, F. U. da; BORGES, J. C. da S. Uso dos mapas conceituais como ferramenta de avaliação qualitativa, com ênfase no ensino de Física.

HOLOS, v. 3, p. 186-200, 2018.

DOWNAR, J.; BHATT, M.; MONTAGUE, P. R. Neural Correlates of Effective Learning in Experienced Medical Decision-Makers. PLOS One, v. 6, n. 11, e27768, 2011. 
FRIEDERICI, A. D. et al. The role of left inferior frontal and superior temporal cortex in sentence comprehension: localizing syntactic and semantic processes. Cerebral Cortex, v. 13, p. 170-177, 2003.

GAZZANIGA, M. S. et al.Neurociência cognitiva: a biologia da mente. Porto Alegre: Artmed, 2006.

HATTIE, J. AC.; DONOGHUE, G. M. Learning strategies: a synthesis and conceptual model. Science of Learning, v. 1, n. 16013, p. 1-13, 2016.

JOHNSON, J. A., ZATORRE, R. J. Neural substrates for dividing and focusing attention between simultaneous auditory and visual events. Neuroimage, v. 31, n. 4, p. 1673-1681, 2006.

KANDEL, E. R. et al. Princípios de neurociências. 5ed. Brasil: MCGRAW-HILL, 2014.

KAPUR, M. Examining productive failure, productive success, unproductive failure, and unproductive success in learning. Educational Psychologist, v. 51, n. 2, p. 289-299, 2016.

LENT, R. Cem bilhões de neurônios. 2ed. Rio de Janeiro: Atheneu, 2010.

MATLIN, M. W. Psicologia Cognitiva. 5a ed. RJ: LTC Livros Técnicos e Científicos, Editora S.A., 2004.

MAYER, R. E. Cognitive Theory of Multimedia Learning. In: MAYER, R. (Ed). Cambridge Handbook of Multimedia Learning. Cambridge: Cambridge University Press, 2014.

NOVAK, J. D. Learning, creating and using knowledge: concept maps as facilitative tools in schools and corporations. 2a Ed. New York: Routledge. 2010.

POSNER, M. I.; PETERSEN, S. E. The attention system of the human brain. Annual Review of Neuroscience, n. 13, 25-42, 1990. 
SANTOS, F. S. et al. Interlocução entre neurociência cognitiva e aprendizagem significativa: uma proposta teórica para o ensino de genética. Revista Brasileira de Ensino de Ciências e Tecnologia, v. 9, n. 2, p. 149-182, 2016.

SCHACTER, D. L., BUCKNER, R. L. Priming and the brain. Neuron, v. 20, n. 2, p. 185-195, 1998.

STEVENS, C.; BAVELIER, D. The role of selective attention on academic foundations: A cognitive neuroscience perspective. Developmental Cognitive Neuroscience, 1, 30-48, 2011.

SUMMERFIELD, C., EGNER, T. Expectation (and attention) in visual cognition. Trends in Cognitive Sciences, v. 13, n. 9, p. 403-409, 2009.

SWELLER, J. Cognitive Load Theory. In: SWELLER, J.; AYRES, P. L.; KALYUGA, S. (Orgs.). Psychology of Learning and Motivation. Cap. 2. New York: Springer, p. 37-76, 2011.

VERDI, M. P. et al. Organized Spatial Displays and Texts: Effects of Presentation Order and Display Type on Learning Outcomes. The Journal of Experimental Education, v. 65, n. 4, p. 303-317, 1997.

ZARPELON, E.; RESENDE, L. M. M.; PINHEIRO, N. A. M. Uso de mapas conceituais na disciplina de Cálculo Diferencial 1: uma estratégia em busca da aprendizagem significativa. Revista Brasileira de Ensino de Ciências e Tecnologia, v. 8, n. 2, p. 176-194, 2015.

Recebido: 24 jan. 2019.

Aprovado: 28 mar. 2020.

DOI: 10.3895/rbect.v13n2.9421

Como citar: SILVA, K. S.; FONSECA, L. S.; CORREIA, P. R. M. Abordagem neurocognitiva de processos atencionais envolvidos na aprendizagem mediada por mapas conceituais. Revista Brasileira de Ensino de Ciência e Tecnologia, Ponta Grossa, v.13, n. 2, p. 247-268, mai./ago. 2020. Disponível em:

<https://periodicos.utfpr.edu.br/rbect/article/view/9421>. Acesso em: XXX

Correspondência: Paulo Rogerio Miranda Correia - prmc@usp.br

Direito autoral: Este artigo está licenciado sob os termos da Licença Creative Commons-Atribuição 4.0 Internacional. 